\title{
From metagenomics to the metagenome: Conceptual change and the rhetoric of translational genomic research
}

\author{
ERIC JUENGST AND JOHN HUSS ${ }^{1}$
}

\begin{abstract}
As the international genomic research community moves from the tool-making efforts of the Human Genome Project into biomedical applications of those tools, new metaphors are being suggested as useful to understanding how our genes work - and for understanding who we are as biological organisms. In this essay we focus on the Human Microbiome Project as one such translational initiative. The HMP is a new 'metagenomic' research effort to sequence the genomes of human microbiological flora, in order to pursue the interesting hypothesis that our 'microbiome' plays a vital and interactive role with our human genome in normal human physiology. Rather than describing the human genome as the 'blueprint' for human nature, the promoters of the HMP stress the ways in which our primate lineage DNA is interdependent with the genomes of our microbiological flora. They argue that the human body should be understood as an ecosystem with multiple ecological niches and habitats in which a variety of cellular species collaborate and compete, and that human beings should be understood as 'superorganisms' that incorporate multiple symbiotic cell species into a single individual with very blurry boundaries. These metaphors carry interesting philosophical messages, but their inspiration is not entirely ideological. Instead, part of their cachet within genome science stems from the ways in which they are rooted in genomic research techniques, in what philosophers of science have called a 'tools-totheory' heuristic. Their emergence within genome science illustrates the complexity of conceptual change in translational research, by showing how it reflects both aspirational and methodological influences.
\end{abstract}

\section{Introduction}

"Thus, it seems appropriate to consider ourselves as a composite of many species human, bacterial, and archaeal - and our genome as an amalgam of human genes and the genes of our microbial 'selves'."

"Step forward into the world of metagenomics and we start to see ourselves as supraorganisms whose genome evolved with associated microbial genomes (the microbiome)." 3

"This study supports the theory that we are in fact 'superorganisms' whose metabolism integrates microbial and human features." 4

"We are superorganisms," or so claims virtually any scientific paper one might pick up on the Human Microbiome Project (HMP) and its implications for medicine. In some ways this holist language is quite surprising. After all, metagenomics - a suite

Genomics, Society and Policy, Vol.5, No.3 (2009) ISSN: 1746-5354

(C) ESRC Genomics Network. 
of methods for bulk sequencing the microbial DNA in environmental samples would at first glance appear to be the epitome of reductionism: it ignores as noise everything but molecular genomic information in seeking to solve biological problems . Moreover, the progenitor of the HMP, the Human Genome Project, was famous for its essentialistic promotional metaphors, ${ }^{5,6}$ and even today many interested in "translating" genomic science into practical applications depend on rhetoric that reduces human identity to our genes. ${ }^{7}$ So how is it that the holist concept of the superorganism, with all its spatial and causal indeterminacies, has become ubiquitous as scientists seek to apply genomics to medical subdisciplines like gastroenterology and dermatology?

In this essay, we suggest three explanations that emerge from a close reading of the HMP literature, as hypotheses for further empirical research. The first is the social influence of new scientific partners. Microbial ecologists and microbiologists of the gastrointestinal tract have long held that the human microbiota - the set of microbes that live in and on us - play an important role in human health, at least as important as that of our 'human' cells. By embracing genomic approaches, these scientists gain a powerful platform for promoting their conceptual framework as an antidote to the essentialism of the Human Genome Project. Moreover, the microbiologists' ecological thought-style is attractive to the human genomics community, because it provides the concepts they need in order to address their political obligation to translate genome science into clinical health care benefits. Simply put, one way to make the (famously inaccessible) human genome more manipulable for therapeutic ends is to redefine it to include elements we can manipulate, and by doing so genomic research can live up to its "translational" promise to repay public investments with health benefits. Neither of these first two factors are surprising sources of rhetorical innovation, given what science studies teaches us about the role of political and social forces in scientific conceptual change. The third, however, is of more potential interest, especially for those concerned with the role of metaphor in science. This is the influence of what philosophers of science call the 'tools-to-theory' heuristic: i.e., the ways in which the techniques and operational strategies of a science can serve as models for the phenomena it describes. In this case, metagenomic sequencing tools designed to address ecologically-framed questions have generated a new theoretical entity for genomics - the 'metagenome'. Cumulatively, these three influences expand the HMP scientists' rhetorical repertoire, significantly changing the metaphors for health, disease, and human nature that they use to articulate the meaning of their work.

\section{DNA sequencing as a 'platform technology" for human microbial ecology}

Forty years before the HMP, gastroenterology was revolutionized by the reconceptualization of the human gut as an ecosystem and the application of field ecology theories to the study of gut microbiota. ${ }^{8}$ It became standard, for example, to classify microbial species in the gut as indigenous on non-indigenous (or allochothonus) members of a 'community' occupying a given "habitat" within the gut, to describe the factors that governed successful microbial 'colonization' of the lumen and mucosa in the newborn, and to talk about the "ecological succession to a climax

Genomics, Society and Policy, Vol.5, No.3 (2009) ISSN: 1746-5354

(C) ESRC Genomics Network. 
community" of gut microbiota in the human adult. ${ }^{9}$ This paradigm allowed medical microbiologists to connect their work with that of their colleagues in soil and water microbiology to create "microbial ecology" as a robust field that shares tools and concepts across widely disparate domains. ${ }^{10}$

One of the core problems for this research program in gastroenterology has been distinguishing indigenous from alien microbial species. To do so was important since the assumption was that in a well-functioning gastrointestinal ecosystem, each habitat is inhabited by a community of one or more indigenous microbial species. When the ecosystem has been perturbed and a habitat has been vacated by its native species, alien species may colonize the vacated habitat - the system is 'out of balance'. In such cases, it would be easy for someone studying the system to think that allochothonus species, because they do occupy a niche, are indigenous. As a result, the central tasks for those studying the microbial ecology of the human gastrointestinal tract have been to identify the various habitats, describe the taxonomic composition of the communities that occupy each of these habitats and unravel the interactions between the species in the community and their host both in the climax community and in the successional stages leading up to it. Traditionally, the biggest hurdles in this effort have been sampling the innermost recesses of the intestines and culturing anaerobic bacteria and archaea in vitro. Despite improvements in selective culturing techniques, culturing bacteria and archaea is a painstaking process, and for many communities of interest, the vast majority of microbial 'species' have not been cultured, thus hampering the abilities of microbial ecologists to model the precise contributions of various microbial taxa to the ecological dynamics and trophic exchanges of the particular microbial ecosystem under study. ${ }^{11}$

While human bacteria like E. coli were, as "model organisms," the test beds for the development of the DNA mapping and sequencing techniques used by the Human Genome Project, the real applications for genomics in addressing the microbiologists' problems came from the environmental side of the field, where DNA sequencing techniques were applied to undifferentiated soil and water samples in order to identify all the microorganisms involved and study their molecular interactions. ${ }^{12}$ This was called "metagenomic" analysis, simply to indicate that mixed samples of genomes from multiple species were being analyzed. Metagenomic methods bypass the problems of anaerobic bacterial sampling and culturing by simply extracting the DNA en masse from all of the microbes present in an environmental sample like a cubic inch of soil, water, or stool. From there fragments of the environmentally sampled DNA can be inserted (or 'cloned') into vectors, such as Bacterial Artificial Chromosomes (BACs), fosmids, or plasmids. Laboratory bacteria (such as E. coli) can be manipulated to serve as hosts for these vectors. When these laboratory bacteria divide, they replicate not only their own ancestral DNA but also that contained in the vectors. When the set of laboratory host bacteria encompasses all of the DNA sequence extracted from the environmental sample, it is called a DNA library of the sampled microbial community. ${ }^{13}$

Genomics, Society and Policy, Vol.5, No.3 (2009) ISSN: 1746-5354

(C) ESRC Genomics Network. 
Once a metagenomic DNA library has been obtained there are two general sets of methods to study it: sequence-based and function-based. Sequence-based methods are generally concerned with the question: who's there? In sequence-based metagenomics, the focus is on identifying contiguous sequences, often with the intention of putting the pieces together to identify the bacterial 'species' present in the sample. Alternatively, the goal may simply be to document the genetic diversity of the sample, often for purposes of intercommunity comparison. In function-based metagenomics, the focus is on translating all the random bits of DNA into proteins and screening them for enzymatic activity and other capabilities such as antibiotic resistance and vitamin synthesis. ${ }^{14}$

The ability of metagenomic strategies to bypass the traditional bottlenecks in the microbial ecology research program has made bulk DNA sequencing an indispensable 'platform technology' for that field, both for illuminating microbial community composition and for insights into function that knowledge of gene products provides. The promise and proliferation of these techniques beyond gut microbiology to studies of the microbial flora of different regions of the human epidermis, teeth, hair, ears, pharynx, and genito-urinary tracts made it possible to advance the idea of a comprehensive "human microbiome project" that would use genomic tools to study the entire human body as an ecological biome for microbiota. ${ }^{15}$

At the same time, metagenomic sequencing also provided microbial ecologists with another kind of platform as well: a podium from which to introduce genome scientists to their ecological ways of understanding the human body. Almost every article promoting the HMP takes care to point out that "The gastrointestinal tract can be regarded as a very complex ecosystem, because it does not involve solely eukaryotic tissues like in other organs, but involves interplay between food, host cells and microbes." ${ }^{16}$ On this construction, the body's bacteria are not adventitious parasites, but participating species in an ecosystem that can benefit both them and their human host. Thus, "the microbial ecosystem of the mammalian gastrointestinal tract is in a homeostatic relationship with the host's immune system. As expected for co-evolving systems, both microbe and mammal benefit from this symbiotic partnership."17

To some extent, describing the human body as an ecosystem is meant literally: the ecological paradigm in microbiology does make it possible to discern and study human microbiota in ways that use the term ecosystem much as it is used in environmental sciences. However, it is also evident that there are metaphorical ecosystems in play in the HMP promoters' rhetoric, as when the literature begins describing the body in geographical terms, as a landscape. For example, as one scientist writes:

When a new human being emerges from its mother, a new island pops up in microbial space. Although a human lifespan is a blink in evolutionary time, the human island chain has existed for several million years, and our ancestors stretch back over the millennia in a continuous archipelago. ${ }^{18}$

Genomics, Society and Policy, Vol.5, No.3 (2009) ISSN: 1746-5354

(C) ESRC Genomics Network. 
Like geographical island ecosystems, the human body contains many micro-climates and ecological niches across three major zones: its inner lining (the gut), its exterior covering (the skin) and its multiple and very different orifices. Microbiomists speak of the microbial 'tide pools' of the teeth, and the 'rainforest' of the gut, using the strikingly $19^{\text {th }}$ century language of mapping 'largely unexplored' terrain, populated by 'indigenous' species of bacteria. ${ }^{19}$ As the proponents of the HMP say, "Microbes thrive on us: we provide wonderfully rich and varied habitats, from our UV-exposed, oxic and desiccating skin to our dark, wet, anoxic and energy rich gut that serves as a home to the vast majority of our 100 trillion microbial (bacterial and archaeal) partners." 20

By slipping from literal to figurative uses of ecological language, the HMP proponents set the stage for a very different understanding of the human individual than that evoked by traditional genomic metaphors. Far from being 'read-outs' or projections of their internal DNA 'blueprints', human beings are at the very least very busy sites of external activity, as biological hosts to hosts of microbiota. Moreover, even in the ways in which the term 'microbiome' is used in the literature there are hints of something more. On one hand, 'microbiome' is sometimes used to evoke the older ecological term 'biome', which historically referred to a major biotic community characterized by the dominant forms of plant life and the prevailing climate. For example Nicholson and co-workers in their 2005 paper, 'Gut microorganisms, mammalian metabolism and personalized health care', explicitly defined "microbiome" (in their glossary) as "the entourage of associated microflora in a host", ${ }^{21}$ essentially an association of organisms in an environment. On the other hand, the term 'microbi-ome' is also very readily assimilated to all of the other 'omes' that molecular biology has bequeathed us, to wit: the metabolome, proteome, transcriptome, and genome. On this conception, the microbi-ome is simply the genome of some complement of microbes: the human gut microbi-ome, for example. For example, one author writes that: "The collective microbial genome, termed the microbiome, has a coding capacity that vastly exceeds that of the human genome and encodes biochemical pathways that humans have not evolved." 22 Conceived in this way, the human microbi-ome becomes a complementary counterpart to the human genome within a larger set of genes that influence human physiology: the human 'metagenome'. The equivocation between these two senses of 'microbiome' within the HMP literature is indicative of more than a translational difficulty between fields: as we suggest below, it also signals the reification of metagenome as a molecular entity in its own right.

\section{Discussion: Implications for genetic determinism}

In a recent series of papers, Dupré and O'Malley have argued that metagenomics has been helpful in replacing an entity-based view of biology with a process-based one. ${ }^{23,24,25}$ From the point of view of metagenomics, one can begin to think of microbial communities not as a collection of interacting unicellular organisms, but as multicellular organisms - metaorganisms - sharing genetic resources through different lateral transfer processes, and also engaged in a number of coordinated multicellular processes such as quorum-sensing. As Dupré and O'Malley note:

Genomics, Society and Policy, Vol.5, No.3 (2009) ISSN: 1746-5354

(C) ESRC Genomics Network. 


\begin{abstract}
A final crucial point about metaorganisms is that they are paradigmatically dynamic entities and therefore very clear illustrations of the ultimate necessity of a process-oriented approach to biological investigation. None of the entities that constitute organisms, or which organisms constitute, are static. Genomes, cells, and ecosystems are in constant interactive flux: subtly different in every iteration, but similar enough to constitute a distinctive process. $^{26}$
\end{abstract}

If the human genome is also reconceived as merely another component in such an ecosystem, this deals an obvious blow to the old genetic determinism. Far from being the 'master molecule' in our physiology, our nuclear DNA is demoted to simply another set of cellular genomes jostling for influence within us, reacting to and being regulated by, a set of microbial genomes that outnumber them 10 to one. As Dupré and O'Malley point out, "The original human genome sequencing projects were, from this perspective, about only a tiny and unrepresentative complement of our genes."27

This new level of indeterminacy has important implications. First, it has the potential to undermine the social risks that a deterministic view exacerbates. If our insurers and employers appreciate the causal complexity and power of the metagenome, they may be less likely to penalize us for the potential health risks lying dormant in any of its parts. More importantly for metagenomics' status as a "translational" science, however, the new causal complexity opens up possibilities for preventing and manipulating those health risks. In one interesting picture of the utopia waiting beyond the HMP, the project's proponents manage to capture both these virtues:

Our medical insurance cards will contain one chip for our primate genome, and one for our microbiome. As part of the annual physical exam, physicians will take a stool sample to update the microbiome profile. Just as today a rise in blood pressure from one visit to the next signals a risk of developing heart disease, tomorrow changes in the microbiome profile will herald a predisposition to diseases such as obesity. Therapeutic intervention will follow, likely a combination of individualized nutrition, deliberate 'reprogramming' of the microbiota with addition/removal or stimulation of particular lineages or genetic complements within the microbiome, or use of microbial gene products themselves (or their revealed human gene product targets) as part of our 21 st century pharmacopoeia. ${ }^{28}$

It has been popular amongst genome scientists to cast the ethical, legal, and social issues in genome research as time-limited problems caused by the 'therapeutic gap' between our ability to read people's genomes and our abilities to offer effective remedies to any deficits we find there. As genomic medicine catches up with genome science, the argument goes, the issues caused by this awkward interim should evaporate, because it will no longer be in anyone's interests to discriminate on the basis of genotype. ${ }^{29}$ The relatively slow growth of human gene therapy, pharmacogenomic interventions, and other forms of personalized genomic medicine over the last decade has been discouraging for this line of argument - until now. If

Genomics, Society and Policy, Vol.5, No.3 (2009) ISSN: 1746-5354

(C) ESRC Genomics Network. 
interventions as 'easy and efficient' as probiotic inoculations of genetically modified bacteria can be counted amongst the armoury of personalized genomic medicine, then the closing of the therapeutic gap may well be at hand. This would achieve genomics' "translational" ambitions and perhaps relieve ethical, legal and social constrictions in the process.

The second implication of the indeterminacy that comes with an ecological view of the body is even more encouraging for the genomics community: It suggests an increased range of opportunities for therapeutic intervention. This promise is very important to the political justification for the public's investment in genomics as a "translational" science, and that drives a need to find ways to appropriate the microbial ecologists' view for human genomics. In the HMP literature, this takes the form of stressing the interdependence between human individuals' 'primate lineage' genomes and the genes of the microbiota we host, which forms the next step towards the 'superorganism' concept.

\section{Translational genomic research and the clinical imperative}

After floundering with the infelicity (and inaccuracy) of entering a 'post-genomic era', the genome research community in the US now universally calls its new phase that of 'translational genomic research', to capture its goal of using genomic science to develop specific interventions that can be used in clinical and public health settings to benefit human health. The HMP is counted as "translational genomic research" primarily because it attempts to use genomic tools to address a problem of medical interest, by improving our understanding of the role of bacteria in human physiology and disease. One of the challenges that genomics faced before the HMP was that, beyond genetic risk testing and pharmacogenomic drug dosage screening, the prospects of using genomic knowledge to develop therapeutic interventions in the human genome directly were at best remote. If the scope of genomic medicine can be expanded beyond our 'primate lineage' DNA to the genomes of our microbial flora, however, a world of potential clinical manipulations is opened up, making it possible to satisfy the political imperative to promise health benefits from the public's investments in genomic science. Ultimately, the proponents hope to be able to provide both diagnostic tools, using DNA screening to assess a patient's bacterial needs, and more effective and 'individualized' probiotic therapies to address microbial imbalances. Genetically engineering the bacteria we include in these therapies could provide a useful new avenue for delivering pharmaceuticals and nutrients as well, or even for replicating, in a reversible and safer way, what we might attempt to achieve through somatic cell gene therapy.

In order to make this move, however, genomic science needs a conceptual justification for annexing what already exists scientifically and commercially as clinical microbiology and the practice of 'probiotic medicine'. Embracing the microbiologists' ecological rhetoric and building on it to recast the human genome as a multi-part system is critical to that enterprise. Thus, in its manifesto for the HMP, The New Science of Metagenomics, the National Research Council writes:

Genomics, Society and Policy, Vol.5, No.3 (2009) ISSN: 1746-5354

(C) ESRC Genomics Network. 
In the end, it may be possible to view ecosystems themselves as biological units with their own genetic repertoires and to sidestep consideration of individual species. Then, both 'Who is there?' and 'What are they doing?' could be replaced with 'What is being done by the community? 30

In order to justify this move, proponents of the HMP must explain the roles that the microbiota and the microbiome play as community members in the ecosystem of the human body. From a medical point of view, it is particularly important to know how they are involved in health and normal physiology, because if they are simply parasitic pathogens it is too easy to dismiss them as alien and, thus, outside the pale of human genomic science. Thus, the architects of the NIH HMP write in a background paper:

As twenty-first century medicine evolves its focus towards disease prevention, new and better ways of defining our health status are needed. The gut microbiota is an effector and a reporter of many aspects of our normal physiology. Comparisons of germ-free and colonized animals have shown that the microbiota helps regulate energy balance, both by extracting calories from otherwise inaccessible components of our diet and by controlling host genes that promote storage of the extracted energy in adipocytes (2-4). The microbiota directs myriad biotransformations, ranging from synthesis of essential vitamins to the metabolism of the xenobiotics that we ingest and the lipids that we produce). ${ }^{31}$

Moreover, as this list indicates, among these interesting microbiotic contributions to our physiology are interactions with our 'primate lineage' genes. Microbiota help regulate differential gene expression in different tissues and in response to environmental stimuli, effectively extending the capacity of our cells to differentiate and adapt. As humans and their bacteria have co-evolved, this has meant a role for the microbiome even in the 'internally programmed' process of normal growth and development. As Dupré and O'Malley point out in their review:

Particularly striking is the growing understanding that symbiotic bacteria are required for the proper development of many vertebrates. ... In fact, for the majority of mammalian organism systems that interact with the external world - the integumentary (roughly speaking, the skin), respiratory, excretory, reproductive, immune, endocrine, and circulatory systems - there is strong evidence for the coevolution of microbial consortia in varying levels of functional association. ${ }^{32}$

The key to the success of this shared system of genetic regulation is the wide variation that bacterial genomic plasticity provides, which allows the microbial lineages in our metagenome to evolve quickly in the face of selective pressures. As the National Research Council report explains, "The mechanisms for rearranging coding elements within a genome serve as mutational switches, ensuring that as the environment 
changes due to shifts in chemical, physical or biological conditions, there will be variants in the cell population that can flourish." 33

As our microbial genome evolves under environmental pressure, in other words, so do the messages that it conveys to the human genome, affecting the expression, regulation and replication of our primate genes. ${ }^{34}$

By stressing this functional and evolutionary interdependence, proponents of the HMP can begin to cast the human and the microbial genomes as complementary elements of the same genomic system, both of which must be included in any adequate 'translational' account of human genomics. For example, Blaser writes:

The first human genome project has been completed. It is now time to invest in a second human genome project: sequencing the cumulative genomes of our microbiota. This is likely to uncover more indicator organisms, the presence or absence of which indicates important disease trends. Furthermore, harnessing these sophisticated host-adapted organisms to do our bidding could uncover new medical treatments.

This is an important point to establish for the proponents of the HMP, because it allows them to point out that "although the primate lineage component of the human genome is decoded, sequencing of the microbiome is just beginning", 36 and argue in favour of a concerted effort to complete it, since: "Without understanding the inhabitants of the human microbiome and the mutualistic human-microbial interactions that it supports, our portrait of human biology will remain incomplete." 37

\section{Discussion: Implications for concepts of health and disease}

Advocates of the HMP promise that:

Once these [metagenomic] methods are in hand, the epidemiologist of the future can collect metadata to correlate with variability between his subject's microbiomes. The results of epidemiological studies will be translated into therapies. Our medical insurance cards will contain one chip for our primate genome, and one for our microbiome. ${ }^{38}$

What form those therapies will take, however, will depend on how we frame the information contained in those chips. Taking them to reflect the dynamics of an integrated, interdependent ecosystem will have implications for both the diagnosis and the treatment of human maladies, because of the concepts of health and disease this new framework promotes.

Viewing the human body as an ecosystem of co-existing cellular species poses a major challenge to the $19^{\text {th }}$ century doctrine of specific causation and the models of health and disease it supports. In both Pasteur's germ theory and Virchow's cellular pathology, diseases were understood to be reducible to real things in the world: the pathogens or lesions which could provide necessary and sufficient targets for

Genomics, Society and Policy, Vol.5, No.3 (2009) ISSN: 1746-5354

(C) ESRC Genomics Network. 
intervention. Under this view, diseases are separable from the patients that suffer them; they are understood best as predators attacking the patient, either as invading germs or as devouring wounds. On this model, the proper target for therapeutics is not the epiphenomenal clinical symptoms of the disease, but whatever the disease 'agent' does to cause those symptoms: the infection, the metastasis, or the break. The great successes of the public health movement in combating infectious disease in the early 20th century, and the reorientation of psychiatry to look for the 'organic' bases of mental illness during the same period, owe much to this interpretation of disease, as does the common correlative view that health is largely a matter of being 'clean' and 'whole'.

If the human body is essentially an ecosystem, however, the notions of 'purity', 'integrity' and 'wholeness', on one hand, and 'infection', 'contagion' and 'corruption' on the other, make little sense, since ecosystems are understood to have fluid boundaries and to support multiple species in a cycle of growth, predation and decay. As a result, the microbiomists point out, the metaphors of war no long apply so well to our understanding of health and disease:

In the face of these challenges, the metaphor of "war" on infectious diseases - characterized by the systematic search for the microbial "cause" of each disease, followed by the development of antimicrobial therapies - can no longer guide biomedical science or clinical medicine. A new paradigm is needed that incorporates a more realistic and detailed picture of the dynamic interactions among and between host organisms and their diverse populations of microbes, only a fraction of which act as pathogens. ${ }^{39}$

Of course, ecosystems can have problems, if they are forced into crises by changing contextual conditions. Species can overpopulate, resources evaporate, and interdependent processes collapse.

As HMP promoters emphasize:

Any ecosystem needs to be in a state of balance in order to function effectively. Disturb one component, and dire consequences to the entire system can follow. In nature, ecosystem disruption often results in massive death of the constituent species. The ecosystem we will focus on in this mini-review is the human gut with its extensive and complex flora of microorganisms. The human intestine is part of a truly amazing ecosystem that is essential for the successful and efficient absorption of nutrients. ${ }^{40}$

This rhetoric of balance resonates well with the traditional philosophical competition for the doctrine of specific causation: the old ideas of Galenic humoral pathology and the 'constitutional pathology' that followed it at the end of the 19th century. On this model, which the microbiomists adopt in ecological form, human health is a matter of having one's physiological processes and predispositions (or dyscrasias) aligned correctly to promote homeostasis, so that the body runs as much like a self-regulated and self-sustaining system as possible. As Dupré and O’Malley suggest, "Indeed, it

Genomics, Society and Policy, Vol.5, No.3 (2009) ISSN: 1746-5354

(C) ESRC Genomics Network. 
may turn out that diseases caused by microbial pathogens are best seen not so much as an invasion by a hostile organism, but rather as a kind of holistic dysfunction of the microbiome." ${ }^{41}$

On this model, there are no diseases in an ontological sense; only sick patients whose bodily processes have gone awry in one direction or another. Thus, while infecting patients with new germs seems a counter-intuitive form of treatment against the doctrine of Specific Causation which gave us antibiotics, repopulating a depleted stock of commensal organisms through 'probiotic' infusions is entirely plausible under this reframing, in just the same way that restocking a habitat with keystone species makes sense for environmental preservation. Thus:

Individualized medicine will only become truly individualized when all aspects of an individual, human and bacterial alike, can be considered. A potential model is emerging, in which a disruption in the microbiome results in a functional imbalance, contributing to a pathological state (Fig. 1b). Treatments such as drugs, changes in diet or re-seeding efforts could facilitate a return to the steady state between the human body and resident microbiota, thereby restoring the functions of supermetabolism. ${ }^{42}$

Of course, constitutional pathology also has its constitutional weaknesses from a psychosocial point of view, as history has shown us. ${ }^{43}$ First, if sustaining and recovering my health is a matter of controlling the contextual forces that influence my inner homeostasis, this means that much more of my health care becomes my own responsibility. My physicians (and insurers) cannot and should not be expected to police and redress my complete lifestyle. Instead, it is up to me to improve my diet, avoid toxic environments, and ensure that my habits are conducive to sustaining a healthy microbiosphere. This reallocation of responsibility is both 'empowering' for the individual, and potentially exculpatory for the social actors who might ordinarily bear responsibility for health care.

Moreover, an extension of this shift of responsibility is the form of stigmatization that typically accompanies 'balance' models of health and disease: the social perception of an individual as intrinsically inclined to go awry in specific ways. We already know the alcoholic, the presymptomatic victim of Huntington's Disease, and those labeled "at risk" by probabilistic genetic testing. ${ }^{44}$ Now it is possible to be vulnerable to disease by sustaining a maladaptive microbiome as well, like the habitual 'abusers' of antibacterial products who encourage the selection of drug resistance within their indigenous flora.

Finally, as this discussion of health suggests, the metagenomic model also raises questions about who is the beneficiary of health or the victim of disease. As the microbiomists point out: "In addition to being numerous, our microbes also are enormously varied - more than 1,000 bacterial species abound in a variety of niches in our bodies. This immediately raises the question of who we are." 45 By reifying the microbial ecologists' instrumental use of bulk sequencing to create the 'metagenome' 
as a biological entity, the proponents of the HMP are able to advance "a superorganism" as their answer.

\section{The "metagenome" and the human superorganism.}

As important as the sociological and political factors are in framing the unusual rhetoric of the HMP, its most provocative claims about human nature also have important roots in the project's 'metagenomic' methods. The HMP is metagenomic because it seeks to do bulk DNA sequencing of multiple bacterial species, like other metagenomic research projects. Unlike its predecessors, however, the HMP aspires to go further to support studies that include human somatic cell genomes in the metagenomic mix, in order to understand our inter-species interactions at the molecular level. As the US National Research Council explains in its ringing endorsement of the HMP:

The human "metagenome" might be considered an amalgamation of the genes contained in the Homo sapiens genome and in the microbial communities that colonize the body inside and out. The organisms within these communities are collectively known as the human "microbiome." The metagenome of these communities encodes physiological traits that humans have not had to evolve, including the ability to harvest nutrients and energy from food that would otherwise be lost because we lack the necessary digestive enzymes. Without understanding the inhabitants of the human microbiome and the mutualistic human-microbial interactions that it supports, our portrait of human biology will remain incomplete. ${ }^{46}$

As this quote suggests, if the architects of the Human Genome Project hoped to achieve a "slow but steady conceptual evolution" in biomedicine, ${ }^{47}$ the proponents of the Human Microbiome Project have much grander ambitions: they to seek to spark nothing short of Kuhnian scientific revolution in our understanding of human biology. They argue that as the "new science" of metagenomics emerges:

Basic ideas that organize biologists' understanding of the living world may need refinement in the face of greater understanding of [microbial] community function. New concepts of genomes, species, evolution, and ecosystem robustness will have effects beyond the specific field of microbiology. The questions that must be asked are "deep" ones... ${ }^{48}$

At so early a stage it is difficult to tell whether the metagenomics revolution represents a Kuhnian "paradigm shift" or simply provides researchers with a better set of tools with which to work on the traditional problems of microbiology and microbial ecology. Interestingly, there is another, non-Kuhnian mechanism of scientific change that holds promise for understanding the transition of the metagenomics research program from a set of tools to a new ontology and set of concepts: the "tools-totheories heuristic'. Gigerenzer coined the phrase "tools to theories heuristic" to refer to the modelling of theoretical concepts on the very tools and techniques of empirical

Genomics, Society and Policy, Vol.5, No.3 (2009) ISSN: 1746-5354

(C) ESRC Genomics Network. 
investigation. ${ }^{49}$ While new tools are often developed in order to answer old questions that had been heretofore recalcitrant, he notes that those same tools often bring with them possible new ways of conceiving the phenomenon under study. In Gigerenzer's work on the history of cognitive psychology, he found, for example, that researchers using Bayesian statistics to analyze their experimental data on human cognition tended to conclude that their research subjects cognized like Bayesians! A less extreme form the tools-to-theories heuristic might take is for the knowledge products of our empirical tools to become reified as theoretical entities. In the history of intelligence research, for example, we can look to Gould's work on the reification of " $\mathrm{g}$ " as the factor underlying the concept of a unitary "general intelligence factor". ${ }^{50} \mathrm{In}$ looking at the relatively recent, relatively short history of metagenomic research, it appears that a tools-to-theories heuristic may be at work as the techniques of metagenomics have given rise to the 'metagenome', conceived of as a genomic unit in its own right. ${ }^{51}$

Conceptually converting a research strategy - metagenomic sequencing - into a new theoretical entity - the "metagenome" - provides a provocative answer to Blaser's question about "who we are": biologically, at least, we are not intrinsically individuals, but collective "metaorganisms", assimilating multiple species, millions of individual organisms, and billions of genes. As almost every article in this literature argues, seeing our bodies as interdependent ecosystems encourages us to find a way to incorporate our microbiome into our sense of self. "Thus, it seems appropriate to consider ourselves as a composite of many species - human, bacterial, and archaeal and our genome as an amalgam of human genes and the genes of our microbial 'selves'., 52

In order to explain this amalgam, the microbiomists borrow from zoology the concept of the "superorganism" 53 to assert that: "Together with our microbes we are a humanbacterial superorganism with immense metabolic diversity and capacity." The basis for this ambitious claim lies in the radical way in which metagenomics reconceives biological individuality. Traditionally, the individual mammalian organism - the horse or the human - has been the paradigm for both folk and scientific concepts of biological individuality. ${ }^{54}$ In spite of the fact that we mammals are rather atypical of living beings generally, this vision of individuality has structured both our biomedical science and our social practices. By using metagenomics to study human physiology, the HMP challenges these boundaries methodologically, and, by extension, the ontological categories we use to "carve nature at its joints". Far from helping genomics realize the modern scientific project of reducing the ills of human individuals to their specific causes, metagenomics and the HMP seem poised to bring genomics to a post-modern understanding of the individual itself, as a pragmatic construction that we project upon on a much more complex system. ${ }^{55}$ Thus, the NRC concludes that:

All plants and animals, including humans, can be considered superorganisms composed of many species, animal, bacterial, archaeal, and viral. Historically, the study of physiology has not focused on these host-associated microbial communities: 
metagenomics offers an opportunity to understand their physiological role. .... The metagenome of these communities encodes physiological traits that humans have not had to evolve, including the ability to harvest nutrients and energy from food that would otherwise be lost because we lack the necessary digestive enzymes. ${ }^{56}$

\section{Discussion: Implications for human identity}

As the microbiomists acknowledge, "We are just beginning to realize the implications of being a superorganism, and the benefits of better knowing our intestinal inhabitants." ${ }^{, 7}$ Indeed, one of the most tantalizing features of the promotional literature on human metagenomics is the suggestion by the heralds of the HMP that this research has revolutionary implications for our ordinary understanding of human nature and what it means to be a human being. In particular, two issues with significant ethical and social implications seem to be made more challenging by the superorganism conception.

First, a superorganismic anthropology recalls the debates inspired by the philosopher Derek Parfit about the stability of the human individual over time, and the ethical implications of a transient conception of the self. ${ }^{58}$ Our conventional moral commitments to respect for personal autonomy, promise-keeping, truth-telling, and the rights and responsibilities that come with moral agency all assume that human individuals display a continuity of identity over time. Parfit's critique of that continuity has created a philosophical industry in efforts either to rebut his views on behalf of ethics or to show how ethics can still apply across serial selves. If metagenomics suggests that our symbiotic microbial populations are integral to our identities as individual organisms and that they change over environments and time, this will bring Parfit's view to the public in tangible ways, by suggesting that even the most 'personalized' medicine possible will face continuously shifting patients. As Dupré and O’Malley point out:
A final crucial point about metaorganisms is that they are paradigmatically dynamic entities and therefore very clear illustrations of the ultimate necessity of a process-oriented approach to biological investigation. None of the entities that constitute organisms, or which organisms constitute, are static. Genomes, cells, and ecosystems are in constant interactive flux: subtly different in every iteration, but similar enough to constitute a distinctive process. ${ }^{59}$

Mining the literature on the ethical implications of the loss of the 'enduring self' may be an important first step in preparing for the public reception of this paradigm shift.

Second, the superorganism concept has dramatic implications for our notions of the integrity of the human species. The normative importance of 'species integrity' has been posited before within bioethics, to critique biomedical practices as varied as the fertilization of hamster ova with human spermatozoa, the transplantation of porcine organs into humans, the propagation of human stem cell lines in mice and the transfer

Genomics, Society and Policy, Vol.5, No.3 (2009) ISSN: 1746-5354

(C) ESRC Genomics Network. 
of human genes into bacteria. 'Crossing species barriers' sounds like trespassing, and the qualms it provokes have been explained in a variety of ways, from invocations of essentialistic or theological visions of human (and animal) nature to fears about risks of creating new forms of disease or ecological disruptions. Common across these claims, however, is the worry that biomedicine will undermine a given stability in the world by violating the categories that order it. Drawing in equal measure from Aristotelian essentialism and $19^{\text {th }}$ Romantic sensibilities, this concern gives high normative weight to the biological kinds produced by the "Wisdom of Evolution" and their relative ranking in a hierarchical 'great chain of being'. On this view, 'splicing life' in the creation of transgenic organisms, or interspecies tissue chimeras, or hybridized embryos - like importing alien species into an established ecosystem - is always dangerous enough to justify the use of the precautionary principle. For some, as Stout has pointed out, the creation of such "abominations" is also morally suspect, simply in its willful disregard for the natural order it crosses. ${ }^{60}$

Anthropologists suggest that these concerns are often animated by the tacit roles that concepts of 'purity' and 'pollution' play in our cultural definitions of human bodily boundaries, health and disease. ${ }^{61}$ In situations that involve the integrity of the human species, like xenotransplantation, or the creation of man-machine 'cyborgs', this moral hazard can be explained as the danger of dehumanization: that polluting the constellation of traits that humans have inherited from our ancestors - our given 'human nature' - with nonhuman attributes we will inevitably degrade the elements of human identity we find morally important, like human dignity, autonomy, and vulnerability. As Habermas puts it:

What is at stake is a dedifferentiation, through biotechnology, of deep rooted categorical distinctions which we have as yet, in the description we give of ourselves, assumed to be invariant. This dedifferentiation might change our ethical self-understanding as a species in a way that could also affect our moral consciousness. ${ }^{62}$

But meanwhile, we know that on and in the healthy human body microbial cells outnumber human cells 10 to one, and play an active role in maintaining our normal physiology. Now the HMP is underlining the significance of that role by suggesting that it may involve interactions at the genetic level as well, between human and bacterial cells. Our commensal bacteria, in essence, serve as crucial genomic extenders, much as they do in termites (whom they allow to digest wood). If so, the microbiologists argue, our basic concept of the human organism should be expanded to include our normal symbionts. Moreover, since our metagenomic profiles will vary among individuals and wax and wane over time, this science suggests that a canonical set of 'human genes' will never be available as a ground for human rights, or for determining when humans' 'species integrity' has been breached.

No one seems much concerned that we are all mixtures of many indigenous bacterial species and human cell lines. It neither undermines our fundamental rights, nor confers special moral status on bacteria. But it does seem as if concerns about the moral implications of blurring boundaries between the human species and non-human

Genomics, Society and Policy, Vol.5, No.3 (2009) ISSN: 1746-5354

(C) ESRC Genomics Network. 
organisms are animating those who are striving to protect our species' 'dignity and integrity'. If we are already superorganisms, those who are concerned are going to have to look further for an explanation for their anxieties.

\section{Conclusion: A new reductionism?}

In the glare of medicine's 19th century paradigms, it is hard to see what the metagenomic revolution might produce. It is clear, however, that better approaches to the understanding of complex systems will be required to translate its science into practical benefits and its message into public discourse. As the microbiomists say:

The level of complexity required to take a dynamic ecological view of human microbiota is daunting, and will require collaborations among many disciplines including molecular biology, ecology, medicine, epidemiology and mathematics. To fully understand the mechanisms that drive community structure and function, microbiota must be examined over time to determine the dynamics of its processes, and over space to determine the interconnectedness of microbiota within an individual host and the range of microbiota among individuals. ${ }^{63}$

In fact, if these complexities are neglected, the risk remains that the "metagenome" will fall prey to the same kinds of reductionism as individual genomic approaches. For example, Handelsman and her colleagues ${ }^{64}$ say that the historical importance of metagenomics to soil ecology lay in its ability to help microbial ecologists conceive of the entire DNA sequence of the environmental sample as a single genomic unit - the metagenome - enabling it to be cloned into BAC vectors which can be carried by $E$. coli. The BAC clones can then be screened for biological activity and novel gene products. But note that despite the ecological window dressing and air of holism, the tools of metagenomics take the complexity of the microbial ecosystem and reduce it to a single genetic sequence: the metagenome. If we draw a direct parallel, it would seem that to treat the gut microbiome as a genomic unit would downplay the role of ecological interactions and place a greater emphasis on gene product discovery. Therefore, while much of the discussion over the translational implications of metagenomics has centered on holistic notions such as restoring balance to the microbial ecosystem, if the entire system is reduced to its metagenome, it is prone to be turned into a tool for the discovery of traditional pharmacological agents, aimed at specific gene products and microbial variants.

Along these lines, it is worth pointing out that one of the major discoveries of metagenome research has been an increased understanding of the metagenomic basis of anti-microbial resistance. For example, Coque and co-workers ${ }^{65}$ studied the metagenome of an entire hospital in Madrid over an 11-year period and found that bacterial resistance to several antimicrobial agents (due to the presence of extended spectrum $\beta$-lactamases [ESBLs], a family of enzymes) persisted despite the ephemeral nature of the bacterial species responsible for producing these enzymes. They concluded that the genes coding for ESBLs were housed in transposable genetic elements as a minority gene population in the hospital metagenome. Residing in the

Genomics, Society and Policy, Vol.5, No.3 (2009) ISSN: 1746-5354

(C) ESRC Genomics Network. 
hospital metagenome, these 'jumping genes' persisted in a variety of bacterial species that themselves were not persistent, making certain bacterial infections difficult to treat.

In light of this, it is ironic that one of the most promising directions for the translation of metagenome research is said to be the discovery of new antibiotics, a continuation of the war metaphor for host-bacterial interactions that many microbial ecologists had been working to overturn While this is far from the only direction that translational metagenomics research might go (in fact, metagenomics has also been instrumental in discovering novel genes conferring antimicrobial resistance ${ }^{66}$ ) it certainly is one area receiving much attention, particularly because of its commercial prospects.

To the extent that the metaphors of metagenomics accurately capture the philosophical implications of the science, translating the message of microbiomics will require tools for talking about issues of human identity, health and disease, and genetic causation in ways that run counter to the messages that genomics has taught us in the past. For this form of "translational genomic research," it may be more important than ever for the many collaborators involved to include not just the list above, but also those disciplines that specialize in capturing what can otherwise be lost in translation in public discourse: the social sciences, the humanities, and the arts.

\section{Acknowledgements}

The research for this paper was supported the Center for Genetic Research Ethics and Law at Case Western Reserve University, under grant \#P50 HG03390 from the U.S. National Human Genome Research Institute and a Faculty Research Grant from The University of Akron to John Huss. We are grateful to valuable discussions of its themes with our colleagues Nicholas King and Aaron Goldenberg, and to invaluable research assistance from Roselle Ponsaran, all to whom the credit for some of its content, but none of its mistakes, must belong.

\footnotetext{
${ }^{1}$ Department of Bioethics, Case Western Reserve University, Cleveland, Ohio, USA; The University of Akron, Ohio USA. Correspondence to: etj2@case.edu

${ }^{2}$ J.L. Gordon, R. Ley, R. Wilson. 2007. Extending our view of self: the Human Gut Microbiome. Initiative (HGMI). National Human Genome Research Institute, http://www.genome.gov/Pages/Research/Sequencing/SeqProposals/HGMISeq.pdf. Accessed 24 June 2007.

${ }^{3}$ R. E. Ley, R. Knight, and J. Gordon. J. The human microbiome: eliminating the biomedical/environmental dichotomy in microbial ecology, Environmental Microbiology 2007; 9:3-4. ${ }^{4}$ I. Sekirov, and B. Finlay. Human and microbe: united we stand. Nature Medicine 2006; 12:736-737.

${ }^{5}$ D Nelkin. Molecular metaphors: The gene in popular discourse. Nature Reviews: Genetics 2001; 2: 555-559.

${ }^{6}$ C. van der Weele. 2005. Images of the genome: From public debates to biology, and back, and forth. In Current Themes in Theoretical Biology. T..C Reydon and L. Hemerik, eds. Springer: 9-31.
}

Genomics, Society and Policy, Vol.5, No.3 (2009) ISSN: 1746-5354

(C) ESRC Genomics Network. 
${ }^{7}$ A. Nordgren, E. Juengst. Can genomics tell me who I am? Essentialistic rhetoric in direct-toconsumer DNA testing. New Genetics and Society 2009; 28: 157-172.

${ }^{8} \mathrm{R}$. Dubos et al. Indigenous, normal, and autochthonous flora of the gastrointestinal tract. J. Exp. Med. 1965; 122: 67-76.

${ }^{9}$ D.C.Savage. Microbial ecology of the gastrointestinal tract. Ann. Rev. Microbiol. 1977; 31:107-133.

${ }^{10}$ J.M. Lynch and N.J. Poole. Microbial ecology: a conceptual approach. New York: John Wiley and Sons.

${ }^{11}$ E.G. Zoetendal et al. Molecular ecological analysis of the gastrointestinal microbiota: A review. $J N$ 2004; 134: 465-472.

${ }^{12}$ C.S. Riesenfeld et al. Uncultured soil bacteria are a reservoir of new antibiotic resistance genes. Environmental Microbiology 2004; 6: 981-989.

${ }^{13}$ National Research Council (US). 2007. The new science of metagenomics: revealing the secrets of our microbial planet. Washington, DC: National Academies Press.

14 Ibid.

${ }^{15}$ Ibid.

${ }^{16}$ E.G. Zoetendal et al. A microbial world within us. Molecular Microbiology 2006; 59: 1639-1650.

${ }^{17}$ M.H. Saier Jr., N.M. Mansour. Probiotics and prebiotics in human health. JMMB 2005; 10: 22-5.

${ }^{18}$ Ley, Knight and Gordon, op.cit. 3 at p.3.

${ }^{19}$ M.J. Blaser. Who are we? Indigenous microbes and the ecology of human diseases. EMBO Rep 2006; 7: 956-960.

${ }^{20}$ National Research Council, op. cit.13, at p.57.

${ }^{21}$ J.K. Nicholson et al. Gut microorganisms, mammalian metabolism and personalized health care. Nature Reviews Microbiology (advance online publication) 2005; 1152: 1-9.

${ }^{22} \mathrm{M}$. Egert et al. Beyond diversity: Functional microbiomics of the human colon. Trends in Microbiology 2006; 14(2): 86-91.

${ }^{23}$ M. O’Malley and J. Dupré. Towards a philosophy of microbiology. Studies in History and Philosophy of Biological and Biomedical Sciences 2007; 38: 775-779.

${ }^{24}$ J. Dupré and M. O’Malley. Metagenomics and biological ontology. Studies in the History and Philosophy of Biology 2007; 38: 834-846.

${ }^{25}$ A. Powell and J. Dupré. From molecules to systems: the importance of looking both ways. Studies in History and Philosophy of Biological and Biomedical Sciences 2009; 40: 54-64..

${ }^{26}$ Dupré and O’Malley, 23, p. 841.

${ }^{27}$ Ibid. at 840 .

${ }^{28}$ Ley, Knight, Gordon, op.cit. 3, p.4.

${ }^{29}$ L. Hood and L. Rown. 1997. Genes, genomes and society. In Genetic Secrets: Protecting Privacy and Confidentiality in the Genetic Era. M. Rothstein, ed. New Haven: Yale University Press: 21-29.

${ }^{30}$ National Research Council, op. cit. 13, p.29.

${ }^{31}$ Gordon, Ley, Wilson, op. cit. 2, p.1.

${ }^{32}$ Dupré and O'Malley, op. cit. 24, p.840.

${ }^{33}$ National Research Council, op. cit. 13, p.34

${ }^{34}$ Nicholson et al. op. cit. 21, p.437.

${ }^{35}$ Blaser, op. cit. 19, p.960.

${ }^{36}$ Ley, Knight, Gordon, op. cit. 3, p.3

${ }^{37}$ National Research Council, op. cit. 13, p.33

${ }^{38}$ Ley, Knight, and Gordon, op. cit. 3, p.3.

${ }^{39}$ Forum on Microbial Threats - Board on Global Health. 2006. Ending the war metaphor: the changing agenda for unraveling the host-microbe relationship: workshop summary. Washington, DC: National Academies Press.

${ }^{40}$ Saier and Mansour, op. cit. 16, p.22

${ }^{41}$ Dupré and O'Malley, op. cit. 24, p.840.

${ }^{42}$ Sikerov and Finley, op. cit. 4, p.737.

${ }^{43}$ E. Juengst. 1999. Concepts of disease after the Human Genome Project. In Ethics and Values in Health Care on the Frontiers of the Twenty First Century (Philosophy and Medicine Book Series, Volume 62). S. Wear and J. Bono, eds. Dordrecht. Kluwer Publishers: 125-150. 
${ }^{44}$ C. Novas, and N. Rose. Genetic risk and the birth of the somatic individual, Economy and Societ,y 2000; 29: 485-513.

${ }^{45}$ Blaser, op. cit. 19, p. 957.

${ }^{46}$ National Research Council, op. cit. 13, p 38.

${ }^{47}$ J. Watson and R. Cook-Deegan. The Human Genome Project and international health. JAMA 1990; 263: 3322-3324.

${ }^{48}$ National Research Council, op. cit. 13, p.33.

${ }^{49}$ G. Gigerenzer, From Tools to Theories: A Heuristic of Discovery .Cognitive Psychology 1991; 98(2): 254-267.

${ }^{50}$ S.J. Gould. 1981. The Mismeasure of Man. New York: W. W. Norton

${ }^{51} \mathrm{~J}$. Handelsman. Metagenomics is not enough. DNA and Cell Biology 2008; 27(5): 219-221

${ }^{52}$ Gordon, Ley, Wilson, op. cit. 2, p.2.

${ }^{53}$ D.S. Wilson and E. Sober. Reviving the superorganism. Journal of Theoretical Biology 1989; 136: 337-356.

${ }^{54}$ J. Wilson. 1999. Biological individuality: the identity and persistence of living entities. New York: Cambridge University Press.

${ }^{55}$ C. Hauskeller. Genes, genomics and identity: projections on nature. New Genetics and Society 2004; 23: 285-301.

${ }^{56}$ National Research Council, op. cit. 13, p.33.

${ }^{57}$ Sekirov and Finlay, op. cit. 4, p.737.

${ }^{58}$ D. Parfit. 1984. Reasons and Persons. Oxford: Clarendon Press.

${ }^{59}$.Dupré and O'Malley, op. cit. 24, p.841.

${ }^{60}$ J. Stout, 1988. Ethics after Babel: The languages of morals and their discontents. Princeton: Princeton University Press

${ }^{61}$ M. Douglas. 1966. Purity and danger: an analysis of the concepts of pollution and taboo. London: Routledge..

${ }^{62}$ J. Habermas. 2003. The future of human nature. Cambridge, UK: Polity Press.

${ }^{63}$ B. Foxman et al. Conceptualizing human microbiota: from multicelled organ to ecological community. Interdisciplinary Perspectives on Infectious Diseases 2008: 4-8.

${ }^{64} \mathrm{~J}$. Handelsman, et. al. Molecular biological access to the chemistry of unknown soil microbes: a new frontier for natural products. Chemistry \& Biology 1998; 5: R245-R249.

${ }^{65}$ T.M. Coque et al. Genes encoding TEM-4, SHV-2, and CTX-M-10 extended-spectrum betalactamases are carried by multiple Klebsiella pneumoniae clones in a single hospital (Madrid, 1989 to 2000). Antimicrob Agents Chemotherapy 2002; 46: 500-510.

${ }^{66} \mathrm{Cf}$. Riesenfeld, et. al., op. cit. p.11. 